\title{
VERY ACCURATE APPROXIMATIONS FOR THE FACTORIAL FUNCTION
}

\section{NECDET BATIR}

Abstract. We establish the following new Stirling-type approximation formulas for the factorial function

$$
n ! \approx \sqrt{2 \pi} n^{n} e^{-n} \sqrt{n+\frac{1}{6}+\frac{1}{72 n}-\frac{31}{6480 n^{2}}-\frac{139}{155520 n^{3}}+\frac{9871}{6531840 n^{4}}}
$$

and

$$
n ! \approx \sqrt{2 \pi} n^{n} e \sqrt[-n]{n^{2}+\frac{n}{3}+\frac{1}{18}-\frac{2}{405 n}-\frac{31}{9720 n^{2}}} .
$$

Our estimations give much more accurate values for the factorial function than some previously published strong formulas. We also derive new sequences converging to Euler-Mascheroni constant $\gamma$ very quickly.

Mathematics subject classification (2010): Primary: 33B15, 40A25; secondary: 41A60, 57Q55.

Keywords and phrases: Gamma function, factorial function, Stirling formula, psi function, Euler Mascheroni constant, harmonic numbers, inequalities, digamma function.

\section{REFERENCES}

[1] M. Abramowitz, I. A. Stegun, Handbook of Mathematical functions with Formulas, Graphs and Mathematical Tables, Dover, New York, 1972.

[2] N. BATIR, Inequalities for the gamma function, Arch. Math. (Basel), 91 (2008), 554-563.

[3] N. BATIR, Sharp inequalities for factorial $n$, Proyecciones, 27, 1 (2008), 97-102.

[4] F. L. BAUER, Remark on Stirling's formulas and on approximations for the double factorial, Mathematical Intelligencer, 28, 2 (2006), 10-21.

[5] W. BURnside, A rapidly convergent series for log N!, Messenger Math., 46 (1917), 157-159.

[6] C. MoRTICI, An ultimate extremely accurate formula for approximation of the factorial function, Arch. Math(Basel), 93 (2009), 37-45.

[7] C. Mortici, New approximations of the gamma function in terms of the digamma function, Appl. Math. Lett., 23, 1 (2010), 97-100.

[8] C. Mortici, Best estimates of the generalized Stirling formula, Appl. Math. Comput., 215, 11 (2010), 4044-4048.

[9] C. MorTici, A class of integral approximations for the factorial function, Comput. Math. Appl., 59, 6 (2009), 2053-2058.

[10] S. Ramanujan, The last notebook and other unpublished papers, Springer, Berlin, 1988.

[11] W. SCHUSTER, Improving Stirling's formula, Arch. Math., 77 (2001), 170-176. 